\title{
Intracranial Aneurysms Associated with Moyamoya Disease
}

\author{
Ljubisa Borota, Slobodan MARINKOVIC*, Radoslav BAJIC**, \\ and Miroslav KovACEVIC ${ }^{* * *}$
}

\begin{abstract}
Department of Neuroradiology of the Institute of Neurosurgery, "Institute of Anatomy, and ${ }^{* * *}$ Department of Neurology of the Emergency Center, Medical Faculty, University Belgrade, Belgrade, Yugoslavia; **Rontgen AVD, Region Sykehus, Tromso, Norway
\end{abstract}

\begin{abstract}
The authors examined 31 patients with moyamoya disease in Yugoslavia. Among them four intracranial aneurysms were disclosed, two in males and two others in females. The aneurysms, which were present in adult patients only, were diagnosed angiographically. Three of four patients died several days or weeks after onset of hemorrhage. The aneurysms were located within the basal abnormal vascular network, on a distal branch of the posterior cerebral artery, on the top of the basilar artery, and on the posterior branch of the middle meningeal artery. The moyamoya disease on the side of the individual aneurysms was most often stage III or IV.
\end{abstract}

Key words: moyamoya disease, intracranial aneurysm, cerebral artery, subarachnoid hemorrhage, cerebral angiography

\section{Introduction}

Intracranial aneurysms have usually been described in individual patients with moyamoya disease, ${ }^{2,8,12,15,17,20,21)}$ as well as in selected cases or in the groups of, predominantly, Japanese patients. ${ }^{1,4,6,9,19)}$ We present our experience with the intracranial aneurysms in a group of 31 patients with moyamoya disease from Yugoslavia. The incidence, location, and the complications of the disclosed aneurysms, including the characteristics of the moyamoya disease, are discussed.

\section{Materials and Methods}

Thirty-one patients, two children and 29 adults, with moyamoya disease have been treated at the Department of Neurosurgery and Department of Neurology during the last 21 years. The adult patients comprised 14 males (mean age 36.6 years) and 15 females (mean age 36.7 years). All patients underwent neurological and angiographical examination and some underwent computed tomography (CT) or magnetic resonance imaging. The aneurysms were found exclu-

Received April 8, 1996; Accepted August 14, 1996 sively in the adult patients. Lumbar puncture was performed in all of the later patients.

\section{Results}

Four intracranial aneurysms were found in two male and two female patients, a incidence of $12.9 \%$. These patients were aged from 35 to 41 years (mean 38.5 years). The patients are presented separately below.

Case 1: A 35-year-old female complained of headaches for several weeks before admission. Her headache rapidly worsened, and she soon began vomiting and lost consciousness. Neurological examination on admission revealed confusion, right-sided hemiparesis, ophthalmoplegia, and signs of meningeal irritation. Lumbar puncture confirmed subarachnoid hemorrhage (SAH). Her condition soon deteriorated and she became comatose. She died 2 weeks later.

Right carotid angiography revealed occlusion of the supraclinoid segment of the internal carotid artery (ICA), as well as the basal abnormal net-like vessels. The posterior cerebral artery (PCA) was filled via the posterior communicating artery ( $\mathrm{PCoA})$. No 


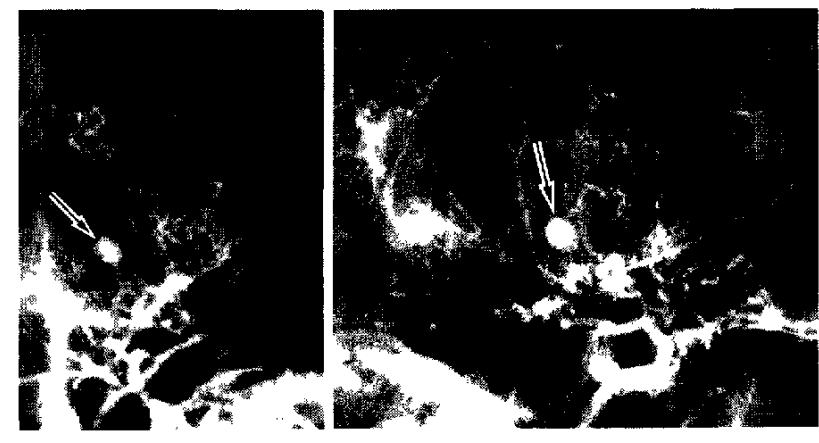

Fig. 1 Case 1. Left carotid angiograms, anterior (left) and lateral (right) views, showing a round aneurysm (arrow) within the basal abnormal vascular network, considered to be a pseudoaneurysm.

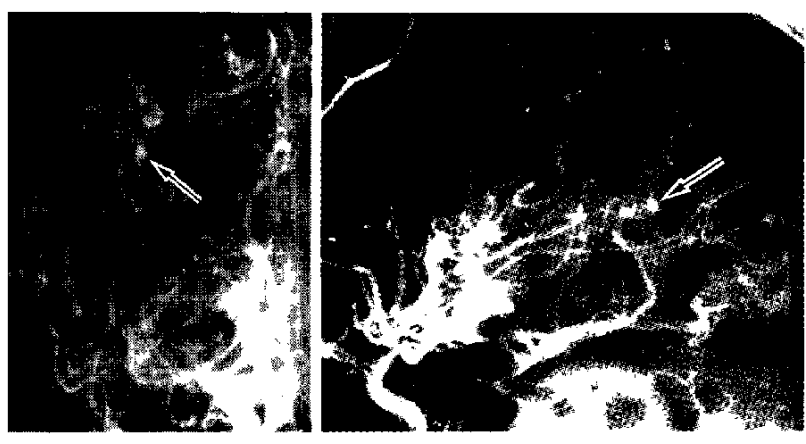

Fig. 2 Case 2. Right carotid angiograms, anterior (left) and lateral (right) views, showing a small aneurysm (arrow) on a distal branch of the PCA.

transdural collaterals were present. Left carotid angiography showed the ICA occlusion, distal to the PCoA origin site. The basal abnormal net-like vessels were dense (stage III), and transdural collaterals were absent. An aneurysm was seen in the basal abnormal vascular network (Fig. 1). The aneurysm was filled from both the anterior (internal carotid) circulation and the posterior (vertebrobasilar) circulation. Case 2: A 39-year-old male complained of headache and then lost consciousness a few days before admission. Neurological examination revealed confusion, disorientation, slight hemiparesis, and facial weakness on the right, as well as signs of meningeal irritation. The cerebrospinal fluid was xantochromic. Psychological examination showed slow thinking, memory disturbance, and emotional change. He gradually recovered and was released soon in good condition.
Right carotid angiography revealed stenosis of the ICA and occlusion of the middle cerebral artery (MCA). The basal abnormal network was clearly seen (stages II-III). PCA was filled via the PCoA. A small aneurysm was disclosed on a distal branch of the PCA (Fig. 2). Left carotid angiography also showed the ICA stenosis, MCA occlusion, and abnormal net-like vessels (stage III). The PCA filled the posterior pericallosal artery.

Case 3: A 39-year-old female was admitted after sudden onset of headache, vomiting, and loss of consciousness. She was comatose on admission. CT revealed diffuse SAH, especially in the basal cisterns. She died 3 days later.

Right carotid angiography showed occlusion of the ICA and reduced basal abnormal vascular network (stages IV-V). The transdural collaterals were developed. Left carotid angiography also revealed the ICA occlusion, reduced basal network, and the transdural collaterals (Fig. 3 left). The branches of the anterior cerebral artery (ACA) and the MCA were filled mainly form the PCA (Fig. 3 center). Vertebral angiography showed a large aneurysm on the top of the basilar artery (Fig. 3 center, right).

Case 4: A 41-year-old male was admitted because of sudden onset of headache and loss of consciousness. Neurological examination showed neck stiffness and bloody cerebrospinal fluid. Right carotid angiography showed ICA occlusion and a reduced abnormal network as well as developed transdural collaterals which filled the branches of the ACA and MCA (stage IV). Left carotid angiography disclosed stenoses of the ICA and ACA, occlusion of the MCA, and reduced basal abnormal network (stages III-IV) (Fig. 4 left). The PCA was mainly filled via the middle meningeal artery. An aneurysm was found on the posterior branch of the middle meningeal artery, which showed very developed transdural anastomoses (Fig. 4 right). Surgery revealed an aneurysm localized partly outside and partly inside of the dura and bloody content in both the subdural and subarachnoid spaces. Despite surgical treatment for this aneurysm, he died 4 days later.

\section{Discussion}

The incidence of intracranial aneurysms in patients with moyamoya disease ranges from $3 \%$ to $14 \% . .^{4,14}$ The incidence in our group of patients was $12.9 \%$. Aneurysms may be associated with moyamoya disease of bilateral or unilateral involvement., ${ }^{2,4)}$ Our patients with aneurysms had bilateral involvement, in stages II to V. ${ }^{18)}$ However, stages III and IV were present most often. 

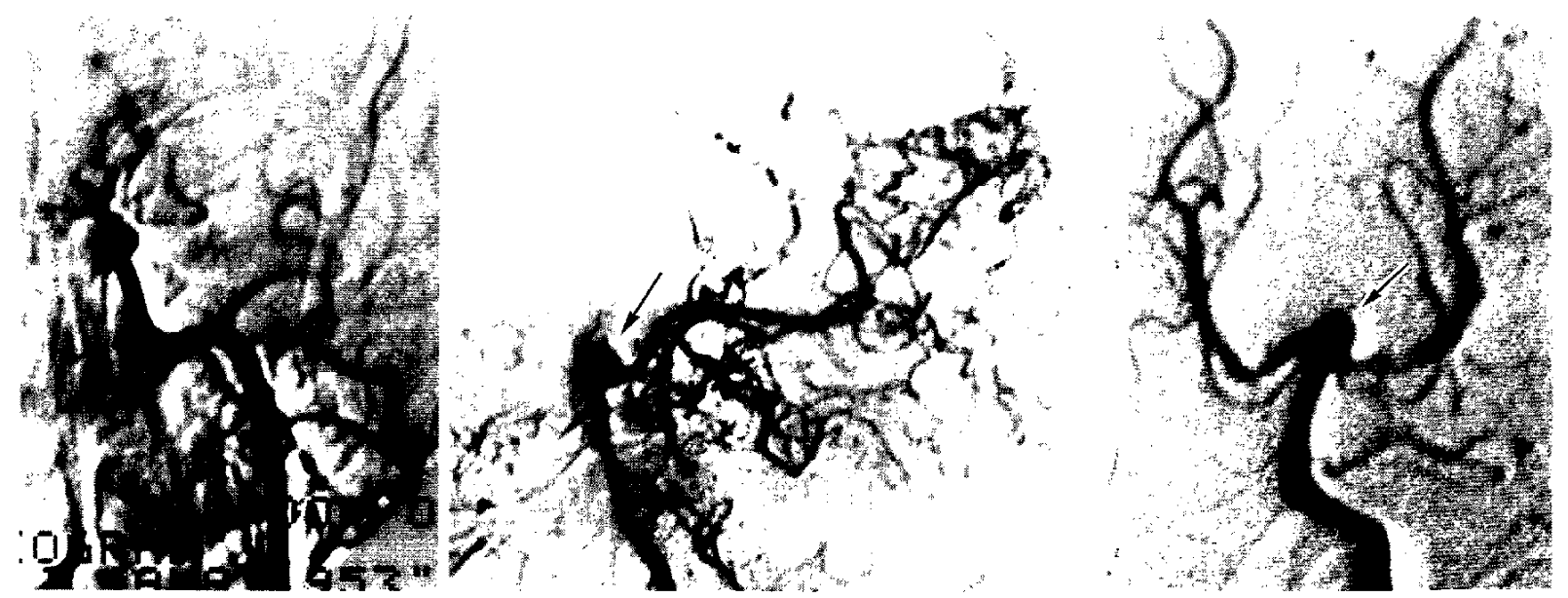

Fig. 3 Case 3. Left carotid angiogram, anterior view (left), showing occlusion of the ICA and reduced basal abnormal vascular network. Left carotid angiogram, lateral view (center), and left vertebral angiogram, anterior view (right), revealing a saccular aneurysm (arrow) on the top of the basilar artery.
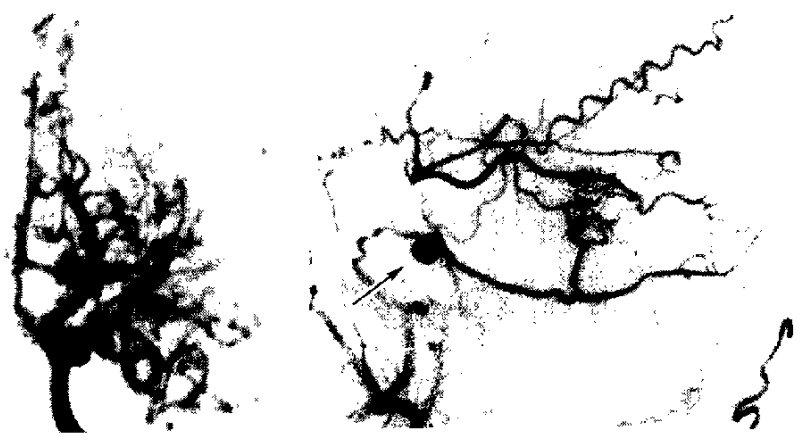

Fig. 4 Case 4. Left carotid angiogram, anterior view (left), showing stenoses of the ICA and ACA and occlusion of the MCA. Left external carotid angiogram, lateral view (right), revealing an aneurysm (arrow) on the posterior branch of the middle meningeal artery, as well as the transdural collaterals.

Aneurysms in moyamoya patients may occur at almost any age from 7 months to 59 years, ${ }^{8)}$ but more often appear in adults than in children ${ }^{7,8,17)}$ and were most often found in adult patients between 36 and 41 years of age. ${ }^{1,5,6,17,19)}$ The mean age of our patients was 38.5 years. Males and females are almost equally represented. ${ }^{1,5,19)}$ A single aneurysm is most common, whereas multiple ones are infrequently present. ${ }^{1,5,9,12,17)}$ No more than two aneurysms have been observed in the same patient. The aneurysms are small, rarely exceeding $10 \mathrm{~mm}$ in diameter ${ }^{99}$ and usually measure between 2 and $10 \mathrm{~mm}$ in diam- eter. ${ }^{5,6,12)}$ Pseudoaneurysms and true aneurysms may occur in patients with moyamoya disease. True aneurysms may be the saccular, fusiform, or dissecting subtype, ${ }^{9)}$ but the last two subtypes occur rarely.

The changes in the parenchymal arteries in moyamoya disease are the pathological basis for the development of pseudoaneurysms. ${ }^{10,11,16,18,21)}$ The changes are mainly due to lipohyalinosis (the hyaline degeneration of the arterial wall), especially fibrocellular thickening of the intima, duplication of the internal elastic lamina, and reduction of the smooth muscle layers of the media. These changes may result in localized dilatation of the parenchymal vessels, i.e. the abnormal net-like vessels (dilated and tortuous perforating arteries), the medullary arteries, and the branches of the anterior choroidal and posterior choroidal arteries. ${ }^{2,6,8,19,21)}$ Pseudoaneurysms are most often located in the abnormal vascular network, especially in the ganglionic and thalamic region (our Case 1), as well as in the periventricular (subependymal) and intraventricular (plexal) regions. Depending on its location, rupture of a pseudoaneurysm may result in intracerebral hematoma and/or ventricular hemorrhage with occasional secondary SAH..$^{2,8,19,21)}$ On the other hand, a pseudoaneurysm can be gradually reduced and may completely disappear. ${ }^{2,5,21)}$

Saccular aneurysms are most likely caused by hemodynamic stress due to increased blood flow and consequent turbulence in certain portions of the cerebrovascular system, especially in the posterior cir- 
culation. ${ }^{1,3,5,6,8,9)}$ The development of aneurysms and/or elastic lamina destruction is especially likely in the presence of a media defect in the wall of the affected arteries. ${ }^{57)}$ Saccular aneurysms are usually located on the main cerebral arteries and their leptomeningeal or choroidal branches, and have been found on the following arteries in moyamoya disease patients: the ICA, i.e. the cavernous $\left(\mathrm{C}_{3}\right)$ segment or the supraclinoid $\left(\mathrm{C}_{4}\right)$ segment (the ophthalmic artery origin), ${ }^{1,11,14)}$ the ACA (the $\mathrm{A}_{2}$ segment, the orbitofrontal or the pericallosal artery origin $)^{4,21)}$; the anterior communicating artery, ${ }^{6,21)}$ the MCA $\left(\mathrm{M}_{1}\right.$ segment), ${ }^{8)}$ the anterior choroidal artery (the origin or distal part), ${ }^{6,19)}$ the PCoA (the origin) ${ }^{1,11,14)}$; the PCA (the $P_{1}$ segment or distal segment) $)^{1,6)}$; the lateral posterior choroidal artery ${ }^{6,12,19)}$; the basilar artery (the distal part or the bifurcation site) ${ }^{1,5,6,8,9,12)}$ and the superior cerebellar artery (the origin). ${ }^{5,6,9)}$ Aneurysms in the moyamoya disease patients are most often located on the bifurcation site of the basilar artery and on the cavernous segment of the ICA ${ }^{6.8 .9)}$ The vertebrobasilar system is involved more often in moyamoya disease patients $(43 \%)$ than in the general population $\left.(5.3-9.6 \%){ }^{8}\right)$ The MCA, ACA, and anterior communicating artery are very rarely involved. ${ }^{8,9)}$

One of our patients had a saccular aneurysm on the middle meningeal artery. Aneurysms on the branches of the external carotid artery are extremely rare whereas this one seems to be the first ever described in a moyamoya patient. Increased blood flow through the meningeal artery and its collaterals was thought to be responsible for development of this aneurysm. Rupture of such aneurysms or potential microaneurysms of the transdural collaterals may cause the epidural hemorrhage, subdural hemorrhage, and/or the SAH. ${ }^{13,14)}$

The results of our study show that the frequency of aneurysms associated with moyamoya disease in $\mathrm{Yu}-$ goslavia seems to be relatively high, even in comparison with data published by Japanese authors. However, the number of patients is too small to perform any serious statistical analysis and obtain reliable results. We found all the most frequent types of aneurysms that may be associated with moyamoya disease in our group of patients. Moreover, we discovered a very unusual type of aneurysm located on the posterior branch of the middle meningeal artery. This finding may contribute to better understanding of the spontaneous collateral blood flow in patients with moyamoya disease.

\section{Acknowledgments}

We thank Professor Masashi Fukui and Assistant Professor Kiyonobu Ikezaki, Department of Neurosurgery, Neurological Institute, Faculty of Medicine, Kyushu University, for their helpful suggestions and preparation of the manuscript.

Also, we thank the Matsumae International Foundation for financial support of work and stay of Ljubisa Borota in Japan (Grant No. 96016).

\section{References}

1) Adams HP, Kassel NF, Wisoff HS, Drake CG: Intracranial saccular aneurysm and moyamoya disease. Stroke 10: 174-178, 1979

2) Grabel JC, Levine $M$, Hollis $P$, Ragland R: Moyamoya-like disease associated with a lenticulostriate region aneurysm. Case report. $J$ Neurosurg 70 : 802-803, 1989

3) Ikezaki K, Matsushima T, Kuwabara Y, Suzuki SO, Nomura T, Fukui M: Cerebral circulation and oxygen metabolism in childhood moyamoya disease: A perioperative positron emission tomography study. $J$ Neurosurg 81: 843-850, 1994

4) Inoue $R$, Katayama $S$, Kasai $N$, Hori S: Middle cerebral artery occlusion with unilateral moyamoya like vessels and with ruptured anterior cerebral artery aneurysm. Its relation to the antiphospholipid antibody syndrome. No To Shinkei 46: 995-998, 1994 (in Japanese)

5) Kodama N, Suzuki J: Moyamoya disease associated with aneurysm. $J$ Neurosurg 48: 565-569, 1978

6) Konishi $Y$, Kadowaki C, Hara M, Takeuchi K: Aneurysms associated with the moyamoya disease. Neurosurgery 16: 484-491, 1985

7) Kono S, Oka K, Sueishi K: Histopathologic and morphometric studies of leptomeningeal vessels in moyamoya disease. Stroke 21: 1044-1050, 1990

8) Kowada $M$, Momma F, Kikuchi K: Intracranial aneurysms associated with cerebrovascular moyamoya disease. Report of a case and review of 13 cases. Br J Radiol 52: 236-237, 1979

9) Massoud TF, Guglielmi G, Vinuela F, Duckwiler GR: Saccular aneurysm in moyamoya disease: Endovascular treatment using electrically coils. Surg Neurol 41: 462-467, 1994

10) Masuda J, Ogata J, Yutani C: Smooth muscle cell proliferation and localization of macrophages and $\mathrm{T}$ cells in the occlusive intracranial major arteries in moyamoya disease. Stroke 24: 1960-1967, 1993

11) Mauro AJ, Johnson ES, Chikos PM, Alvord EC: Lipohyalinosis and miliary microaneurysms causing cerebral hemorrhage in a patient with moyamoya. A clinicopathological study. Stroke 11: 405-412, 1980

12) Nagamine $Y$, Takahashi S, Sonobe M: Multiple in- 
tracranial aneurysms associated with moyamoya disease. Case report. $J$ Neurosurg 54: 673-676, 1981

13) Nakakita $K$, Tanaka $S$, Fukuda A, Fujii $C$, Kohama $A$, Miyasato $H$ : Nontraumatic acute subdural hematoma caused by the rupture of transdural anastomotic vessels in moyamoya disease. No Shinkei Geka 22: 561-565, 1994 (in Japanese)

14) Oppenheim JS, Genusso R, Sacher M, Hollis P: Acute atraumatic subdural hematoma associated with moyamoya disease in an African-American. Neurosurgery 28: 616-618, 1991

15) Picard L, Andre JM, Roland J, Arnould G, Lepore AJ, Crouzet G, Djinjijan R: "Moyamoya" syndrome of the adult transient form. $J$ Neuroradiol 1 : 69-86, 1974

16) Picard L, Floquet J, Andre JM, Montaut J, Salamon $\mathrm{G}$ : The moyamoya syndrome: Anatomico-pathological study. J Neuroradiol 1: 113-132, 1974

17) Satoh S, Shibuya H, Matsushima Y, Suzuki S: Analysis of the angiographic findings in cases of childhood moyamoya disease. Neuroradiology 30: 111-119, 1988

18) Serdaru M, Gray F, Merland JJ, Escourolle R, Grumbach R: Moyamoya disease and intracerebral hematoma. Clinical pathological report. Neuroradiology 18: 47-52, 1979

19) Tanaka $Y$, Ogashiwa $M$, Takeuchi $K$ : The moyamoya phenomenon with accompanying intracranial aneurysm. Neuroradiology 16: 289-290, 1978

20) Yasargil MG, Smith RD: Association of the middle cerebral artery anomalies with saccular aneurysm and moyamoya disease. Surg Neurol 6: 39-43, 1976

21) Yuasa $\mathbf{H}$, Tokito $\mathrm{S}$, Izumi $\mathrm{K}$ : Cerebrovascular moyamoya disease associated with an intracranial pseudoaneurysm. Case report. J Neurosurg 56: 131134,1982

Address reprint requests to: $\mathrm{L}$. Borota, M.D., Department of Neuroradiology, Institute of Neurosurgery, Visegradska 26, Belgrade, Yugoslavia.

\section{Commentary}

This is an interesting paper reporting moyamoya disease in Yugoslavia. The incidence of the aneurysm associated with the disease seems relatively high in Yugoslavia, although it is not clear that all the moyamoya cases are typical. The authors claim that one of their aneurysms occurred in the extracerebral meningeal artery, and is the first such case reported. As moyamoya disease is encountered relatively often in Asian populations, further information as to the incidence, treatment, and follow-up in Yugoslavia or other European countries is awaited.

Shigeaki KOBAYASHI, M.D.

Department of Neurosurgery Shinshu University School of Medicine Nagano, Japan

This is an important contribution depicting a well known, but rare complication of a very uncommon disease.

The authors describe a $13 \%$ incidence in angiographically documented intracranial aneurysms related to patients with moyamoya disease. The incidence reported in the literature ranges between $3 \%$ and $14 \%$, but in that all of the series reported are small, the true incidences remain uncertain. For example, in our group of 39 patients with moyamoya disease, we discovered 12 patients $(30 \%)$ with intracranial aneurysm. This undoubtedly is a reflection of the bias of our referral base and is extraordinarily high.

The importance of this report is in three areas:

(1) A reminder that intracranial aneurysms may be the source of intracranial bleeding, both into the intracerebral and subarachnoid spaces in patients with moyamoya disease, and deserve to be treated like any other aneurysm.

(2) The surgical treatment of these aneurysms may be quite complex because of their unusual and often distal location on the posterior cerebral circulation. Surgeons must take great care and remember the importance of preserving collateral flow during the exposure and clipping of the aneurysm.

(3) The re-affirmation that hemodynamic flow stresses are an important factor in the development of aneurysms. The demonstration of an aneurysm arising from the middle meningeal artery in a patient with spontaneous transdural anastomosis even further reinforces this theory.

Sydney J. Peerless, M.D., F.R.C.S.C. Mercy Neuroscience Institute Florida, U.S.A. 